\title{
A dynamical model of TGF- $\beta$ activation in asthmatic airways
}

Hannah J. Pybus*1, Reuben D. O’Dea*2, Bindi S. Brook*3

\author{
Affiliation: *School of Mathematical Sciences, University of Nottingham, Nottingham, United \\ Kingdom \\ E-mail: ${ }^{1}$ hannah.pybus@nottingham.ac.uk (corresponding author) \\ E-mail: ${ }^{2}$ reuben.odea@nottingham.ac.uk \\ E-mail: ${ }^{3}$ bindi.brook@nottingham.ac.uk
}

$28^{\text {th }}$ March 2021

\begin{abstract}
Excessive activation of the regulatory cytokine transforming growth factor $\beta$ (TGF- $\beta$ ) via contraction of airway smooth muscle (ASM) is associated with the development of asthma. In this study, we develop an ordinary differential equation model that describes the change in density of the key airway wall constituents, ASM and extracellular matrix (ECM), and that accounts for subcellular signalling pathways that lead to the activation of TGF- $\beta$. We identify bi-stable parameter regimes where there are two positive steady states; one with a low concentration of active TGF- $\beta$ and the other with an elevated concentration of active TGF- $\beta$ (and as a result, increased ASM and ECM density). We associate the former with a healthy homeostatic state and the latter with a diseased (asthmatic) state. We demonstrate that external stimuli, that induce TGF- $\beta$ activation via ASM contraction (mimicking an asthmatic exacerbation), perturb the system from the healthy state to the diseased one. We show that the properties of the stimuli, such as the frequency, strength and the clearance of surplus active TGF- $\beta$, are important in determining the long-term dynamics and the development of disease.
\end{abstract}

\section{Introduction}

Despite its rising prevalence in the population, the causes and onset of asthma remain poorly understood. Asthma is a long-term disease of the lungs with the following pathological hallmarks: chronic inflammation, airway hyperresponsiveness and airway remodelling (Brightling et al., 2012; Berair et al., 2013). Airway hyperresponsiveness is defined by exaggerated bronchoconstriction (narrowing of the airway) due to the rapid contraction of airway smooth muscle (ASM) in response to a relatively low dose of contractile agonist (King et al., 1999; West et al., 2013). Swelling of the airway tissue, induced by chronic inflammation, narrows the airway and leads to overall restricted pulmonary function (León, 2017). Airway remodelling comprises the sustained structural changes due to inflammatory injury repair, airway thickening and scarring (Bossé et al., 2008; Al Alawi et al., 2014). Until recently, chronic inflammation has thought to have been the main contributor to airway remodelling (Saglani and Lloyd, 2015). However, current experimental evidence suggests that airway remodelling may be promoted by bronchoconstriction-induced airway narrowing (Grainge et al., 2011).

Bronchoconstriction activates the regulatory cytokine, transforming growth factor $\beta$ (TGF- $\beta$ ) (Wipff et al., 2007; Buscemi et al., 2011; Tatler et al., 2011), which may further stimulate ASM contraction (Ojiaku et al., 2018), ASM proliferation (Chen and Khalil, 2006), and deposition of extracellular matrix (ECM) (Pohlers et al., 2009), altering the airway mechanics (Makinde et al., 2007; Burgess et al., 2016). In the healthy airway, regulatory mechanisms terminate this process (Hinz, 2015), thereby maintaining homeostasis. It is thought that in asthma this homeostatic state is lost, resulting in accumulation of ECM and contraction of ASM, further up-regulating TGF- $\beta$ production through a positive mechanotransductive feedback loop. However, the mechanisms underlying cell-mediated TGF- $\beta$ activation, during an asthmatic exacerbation, are not clear.

The spindle-shaped ASM cells form bundles that surround the lumen of the bronchi (Kuo et al., 2003; Ijpma et al., 2017) and are primarily responsible for contraction, proliferation, cytokine production and ECM secretion. It is suggested that ASM cells express fewer contractile proteins whilst 
proliferating and therefore generate less contractile force than non-proliferating ASM cells (Bentley and Hershenson, 2008). Hence, the roles of each phenotype differ: the contractile phenotype initiates contraction and the proliferative increases ASM mass. The ECM is a delicate mesh of deposited connective tissue and fibrous proteins (Cheng et al., 2016), surrounding ASM cell bundles, that provides physical support and stability to the lung (Burgess et al., 2016; Khan, 2016). Freyer et al. (2001) report that the ECM constituents fibronectin, laminin and collagen appear to aid the survival of the embedded ASM, which in turn secrete compounds to replenish the ECM to form a positive feedback loop. However, the overall accumulation of ECM does not significantly change over time within the normal healthy airway as the ECM appears to exhibit a balance between its deposition and degradation (Makinde et al., 2007).

Initially identified in human platelets as a protein associated with signalling in wound healing (Assoian et al., 1983), the pro-inflammatory cytokine TGF- $\beta$ plays a vital role in regulating the immune system (Letterio and Roberts, 1998). TGF- $\beta$ is secreted as part of the large latent complex (LLC) that resides in the ECM (Tatler et al., 2011). Cell-mediated stress, in response to cytoskeletal reorganisation or reaction to a trigger, induces conformational changes in the LLC leading to the activation of TGF- $\beta$ (Keski-Oja et al., 2004; Wipff et al., 2007). Herein, as in Gleizes et al. (1997) and Annes et al. (2003), we use the term 'TGF- $\beta$ activation' to denote the separation of TGF- $\beta$ from the LLC and 'latent' indicates its inactive storage. Both the contractile ASM and the ECM are required for the mechanical activation of TGF- $\beta$ (Tatler and Jenkins, 2012, 2015; Tatler et al., 2016); however, the process does not reduce their densities. Significant quantities of latent TGF- $\beta$ are contained within tissues, yet activation of only a small fraction of the latent form generates a maximal cellular response (Annes et al., 2003). Once activated, TGF- $\beta$ binds to cell surface receptors (Derynck and Zhang, 2003; Shi et al., 2011; Aschner and Downey, 2016) to initiates multiple cellular responses including cell contraction, apoptosis, differentiation, migration, proliferation, survival and ECM secretion (Khalil et al., 2005; Perng et al., 2006; Berair et al., 2013; Ojiaku et al., 2018).

TGF- $\beta$ expression is increased within the asthmatic airway (Ojiaku et al., 2018) and correlates with asthma severity (Chen and Khalil, 2006; Al Alawi et al., 2014). Continual exposure to an allergen causes TGF- $\beta$ to stimulate a dysregulated repair process of inflammation-induced injury to the airway by promoting ECM deposition and ASM proliferation which consequently thickens the airway wall (Al Alawi et al., 2014) and increases TGF- $\beta$ secretion (Amrani and Panettieri, 2003). In addition, TGF- $\beta$-mediated ASM contraction may further up-regulate TGF- $\beta$ activation. In light of these findings, and in attempt to combat asthmatic symptoms, it is necessary to investigate the feedback mechanisms responsible for excessive activation of TGF- $\beta$ during an asthmatic exacerbation.

Experiments in vitro are typically reductionist in nature. To understand interactions of complex biochemical and mechanical processes requires integrative, predictive mathematical models. There are various mathematical models that account for cell signalling events based upon experimental data (e.g. Haberichter et al. (2002), Brumen et al. (2005), Croisier et al. (2013)), the majority of which employ the law of mass action to obtain ordinary differential equations (ODEs) that describe the rates of change in constituent density. Chernyavsky et al. (2014) present an ODE model representing ASM phenotype switching between a proliferative and contractile state. In the proliferative state, ASM is assumed to grow logistically which implicitly incorporates spatial constraints. They consider the occurrence of both periodic and irregular exacerbations, following a random Poisson process, in order to investigate the range of possible outcomes an individual may experience, given average airway characteristics. They find that the likelihood of developing moderately to severely remodelled airways is critically dependent on the inflammatory resolution rate and that the individual's history of induced remodelling events and accumulation of successive triggers have a long term impact on the degree of ASM hyperplasia.

Similarly, the morphoelastic model of Hill et al. (2018) explores airway remodelling in response to transient inflammatory or contractile agonist challenges. Consistent with the findings of Chernyavsky et al. (2014), they find that when the inflammation resolution is slow, a small number of exacerbation events may lead to significant and persistent airway remodelling. Their results suggest that the sustained contractility of the ASM observed in asthmatics may be due to either a mechanotransductive feedback loop, insufficient clearance of contractile agonists, or a combination of the two. 


\section{Model development}

As summarised above, active TGF- $\beta$ binds to receptors expressed by proliferating ASM, contractile ASM and other surrounding cells which initiates multiple cellular responses (Derynck and Zhang, 2003; Al Alawi et al., 2014; Aschner and Downey, 2016). These responses include the triggering of ASM proliferation and stimulation of ASM contraction. We develop an ODE model to investigate the activation of latent TGF- $\beta$ and the downstream effects of TGF- $\beta$ binding at the cell-level. The schematic diagram in Figure 1 illustrates the interactions between the key airway wall components, ASM and ECM, and the effects of TGF- $\beta$ activation accommodated by our model. We incorporate density-dependent removal of active TGF- $\beta$ due to TGF- $\beta$-receptor binding events and indicate which ASM phenotype expresses the required receptor and the availability of such receptor via the interaction rates. We assume that the receptors expressed by the proliferating and contractile ASM cells are limited so that the binding rate saturates with the combined density of proliferating or contractile ASM and active TGF- $\beta$. This leads to the following system of ODEs describing the rate of change in density of the ASM, ECM and concentration of active TGF- $\beta$ :

$$
\begin{aligned}
\frac{\mathrm{d} p^{*}}{\mathrm{~d} t^{*}} & =\hat{\kappa}_{p}^{*}\left(p^{*}\right) p^{*}\left(1+\hat{\kappa}_{a p}^{*}\left(a^{*}, p^{*}\right)\right)+\kappa_{c p}^{*} c^{*}-\kappa_{p c}^{*} p^{*}, \\
\frac{\mathrm{d} c^{*}}{\mathrm{~d} t^{*}} & =\kappa_{p c}^{*} p^{*}-\kappa_{c p}^{*} c^{*}-\hat{\phi}_{c}^{*}\left(m^{*}\right) c^{*} \\
\frac{\mathrm{d} a^{*}}{\mathrm{~d} t^{*}} & =\kappa_{a}^{*}+\left(\hat{\kappa}_{s}^{*}\left(t^{*}\right)+\hat{\kappa}_{a c}^{*}\left(a^{*}, c^{*}\right)\right) c^{*} m^{*}-\kappa_{b}^{*} a^{*}\left(p^{*}+c^{*}\right)-\phi_{a}^{*} a^{*}, \\
\frac{\mathrm{d} m^{*}}{\mathrm{~d} t^{*}} & =\left(\kappa_{c m}^{*}+\hat{\kappa}_{a c m}^{*}\left(a^{*}, c^{*}\right)\right) c^{*}+\left(\kappa_{p m}^{*}+\hat{\kappa}_{a p m}^{*}\left(a^{*}, p^{*}\right)\right) p^{*} \\
& \quad+\hat{\kappa}_{e}^{*}\left(a^{*}\right)-\phi_{m}^{*} m^{*},
\end{aligned}
$$

where, following Chernyavsky et al. (2014), we assume that the ASM may be divided into two separate sub-populations namely, the proliferative phenotype, $p^{*}\left(t^{*}\right)$, and contractile phenotype, $c^{*}\left(t^{*}\right)$. Furthermore, we assume that phenotype switching may occur between these two ASM sub-populations.

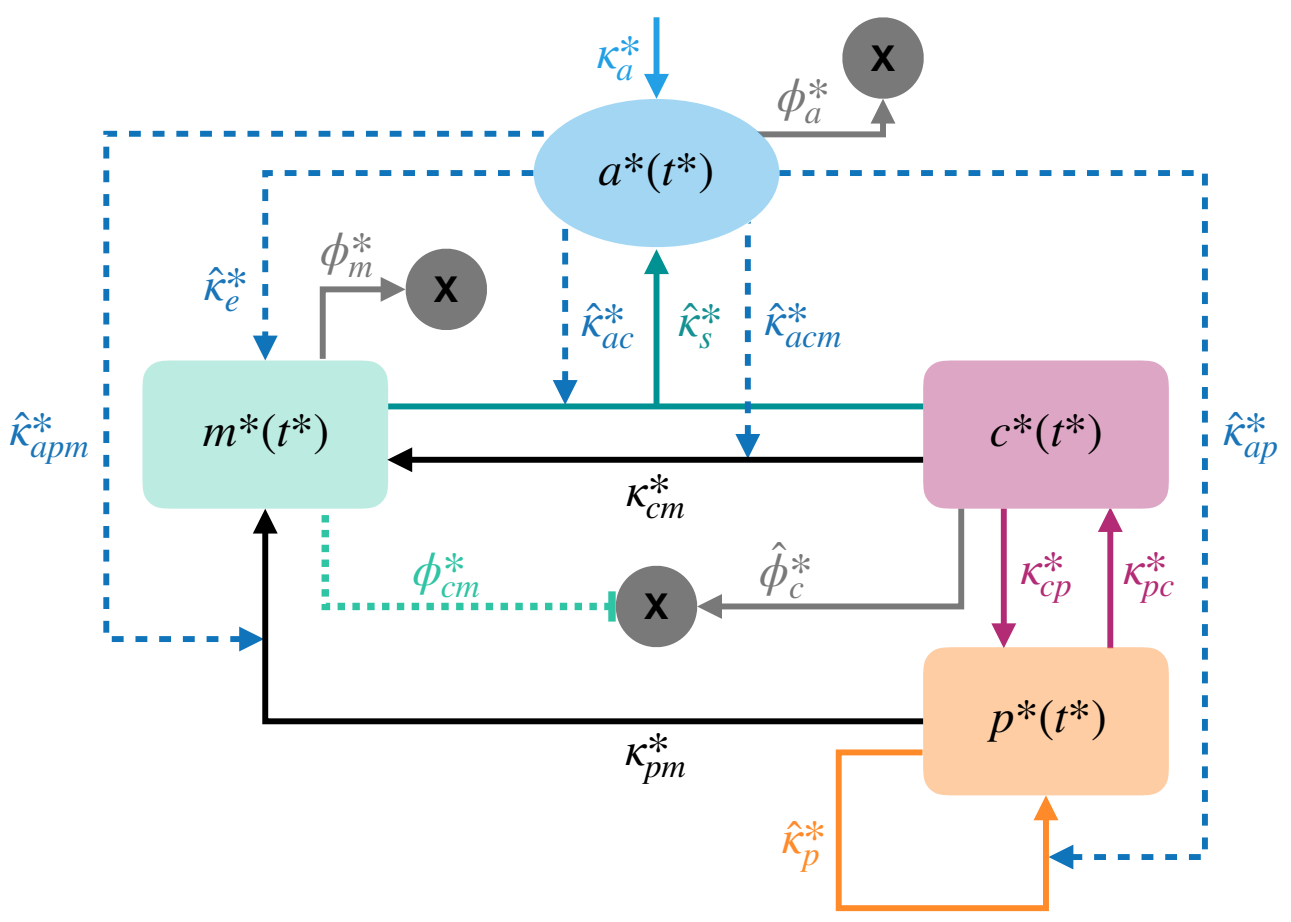

Figure 1: Schematic diagram detailing the interactions between the key components, proliferating ASM, $p^{*}\left(t^{*}\right)$, contractile ASM, $c^{*}\left(t^{*}\right)$, ECM, $m^{*}\left(t^{*}\right)$ and active TGF- $\beta, a^{*}\left(t^{*}\right)$, at rates $\kappa$ and $\phi$ as listed, explained and nondimensionalised in Table 1 in Appendix A. Dashed blue lines represent TGF- $\beta$ signalling pathways and the grey circles, labelled X, represent degradation of each of the respective components. 
As described above in Section 1, the roles of the proliferating ASM and contractile ASM differ, and the contractile ASM phenotype is exclusively responsible for contraction, whereas the proliferative phenotype is exclusively responsible for ASM growth. The rate constant $\kappa_{p c}^{*}$ represents phenotype switching from the proliferative to contractile state and the rate constant $\kappa_{c p}^{*}$ represents phenotype switching from the contractile to proliferative state. We assume that the proliferation rate, $\hat{\kappa}_{p}^{*}\left(p^{*}\right)$, is logistic,

$$
\hat{\kappa}_{p}^{*}\left(p^{*}\right)=\kappa_{p}^{*}\left(1-\frac{p^{*}}{p_{\max }^{*}}\right)
$$

thereby implicitly accounting for a spatial constraint, $p_{\text {max }}^{*}$, defining the carrying capacity of the proliferating ASM cell density. ASM proliferation is thought to increase with asthma severity (Johnson et al., 2001; Trian et al., 2007; Pelaia et al., 2008; Januskevicius et al., 2016) and correspondingly, the rate constant $\kappa_{p}^{*}$, defining the rate of ASM proliferation, may be altered to reflect the severity of asthma.

The rate function $\hat{\kappa}_{a p}^{*}\left(a^{*}, p^{*}\right)$ represents active TGF- $\beta$-induced proliferation and acts in addition to baseline proliferation $\hat{\kappa}_{p}^{*}$. The rate function $\hat{\kappa}_{a c}^{*}\left(a^{*}, c^{*}\right)$ represents activation of further TGF- $\beta$ as a result of active TGF- $\beta$-induced contraction of the ASM. The rate functions $\hat{\kappa}_{a c m}^{*}\left(a^{*}, c^{*}\right), \hat{\kappa}_{a p m}^{*}\left(a^{*}, p^{*}\right)$ and $\hat{\kappa}_{e}^{*}\left(a^{*}\right)$ represent the rate of secretion of ECM from the contractile ASM, proliferating ASM and other surrounding cells, respectively, that is induced by active TGF- $\beta$ signalling pathways. In addition, baseline secretion of ECM (independent of active TGF- $\beta$ concentration) occurs at rates $\kappa_{c m}^{*}$ and $\kappa_{p m}^{*}$ from the contractile and proliferative ASM cells, respectively. Furthermore, it is assumed that the density of proliferating and contractile ASM is not changed as a result of secretion of ECM.

Following the approach of Baker et al. (2017), we assume that rates that depend on the concentration of active TGF- $\beta$ are biologically limited and take the form of saturating Hill functions. The rates for TGF- $\beta$-induced ASM proliferation, $\hat{\kappa}_{a p}^{*}\left(a^{*}, p^{*}\right)$, and ECM secretion, $\hat{\kappa}_{a p m}^{*}\left(a^{*}, p^{*}\right)$, are

$$
\hat{\kappa}_{i}^{*}\left(a^{*}, p^{*}\right)=\frac{\kappa_{i}^{*}\left(a^{*} p^{*}\right)^{n_{p}}}{\tilde{\eta}_{i}^{*}+\left(a^{*} p^{*}\right)^{n_{p}}}, \quad i \in\{a p, a p m\} .
$$

Here, $\hat{\kappa}_{i}^{*}$ is a function of the product $a^{*} p^{*}$ to reflect the necessity of active TGF- $\beta$ binding to receptors expressed by the proliferating ASM cells in order to initiate the associated pathway (e.g. ASM proliferation). Likewise, the rates for active TGF- $\beta$-induced ASM contraction, $\hat{\kappa}_{a c}^{*}\left(a^{*}, c^{*}\right)$, and ECM secretion, $\hat{\kappa}_{a c m}^{*}\left(a^{*}, c^{*}\right)$, are

$$
\hat{\kappa}_{i}^{*}\left(a^{*}, c^{*}\right)=\frac{\kappa_{i}^{*}\left(a^{*} c^{*}\right)^{n_{c}}}{\tilde{\eta}_{i}^{*}+\left(a^{*} c^{*}\right)^{n_{c}}}, \quad i \in\{a c, a c m\} ;
$$

as above, the dependence on $a^{*} c^{*}$ indicates initiation of ASM contraction by TGF- $\beta$-contractile ASM cell binding. We assume that the receptors expressed by other surrounding cells (excluding ASM cells) are in abundance so that the rate for active TGF- $\beta$-induced ECM secretion from external sources, $\hat{\kappa}_{e}^{*}\left(a^{*}\right)$, is

$$
\hat{\kappa}_{e}^{*}\left(a^{*}\right)=\frac{\kappa_{e}^{*} a^{* n}}{\tilde{\eta}_{e}^{*}+a^{* n}} .
$$

ECM presence has been shown to aid the survival of contractile ASM (Freyer et al., 2001). We therefore assume that the apoptosis rate of contractile ASM depends on the ECM density such that

$$
\hat{\phi}_{c}^{*}\left(m^{*}\right)=\phi_{c}^{*}-\frac{\phi_{c m}^{*} m^{* n_{m}}}{\tilde{\eta}_{c m}^{*}+m^{* n_{m}}},
$$

where, in the absence of ECM aided survival, apoptosis of contractile ASM occurs at a maximum rate $\phi_{c}^{*}$. In order to ensure that the presence of ECM does not directly increase the density of ASM, yet reduces the ASM apoptosis rate towards a minimum as the further addition of ECM no longer significantly aids ASM survival, we enforce $\phi_{c}^{*}>\phi_{c m}^{*}$. ECM degrades at rate $\phi_{m}^{*}$. The Hill coefficients $n_{p}, n_{c}, n$ and $n_{m}$ in (3)-(6) represent the order of saturation.

Activation of latent TGF- $\beta$, via alternative sources, is assumed to occur basally (Burgess et al., 2016) at a constant rate $\kappa_{a}^{*}$ in addition to mechanical activation of latent TGF- $\beta$ (Wipff et al., 2007; 
Buscemi et al., 2011). Recall that ASM cell contraction mechanically activates latent TGF- $\beta$ (Tatler and Jenkins, 2012), by unfolding the latent complex that is anchored to the ECM (providing resistance (Hinz, 2015)) and that encapsulates latent TGF- $\beta$. Hence, both the contractile ASM and ECM are required for the mechanical activation of TGF- $\beta$ (Tatler et al., 2011); however it is assumed that the process does not result in the loss of either constituent. Latent TGF- $\beta$ is found in abundance (Annes et al., 2003) and therefore is not a limiting factor in the mechanical activation step. Activation of latent TGF- $\beta$ in response to an external stimulus, $\hat{\kappa}_{s}^{*}\left(t^{*}\right)$, represents exposure to an irritant and mimics an asthmatic exacerbation. Experimentally, the stimulus may represent the administration of a contractile agonist such as methacholine $(\mathrm{MCh})$ that is known to cause contraction of the ASM in lung-slice models (Tan and Sanderson, 2014). Following Chernyavsky et al. (2014), we model this as a series of Gaussian stimuli, given by

$$
\hat{\kappa}_{s}^{*}\left(t^{*}\right)=\frac{\kappa_{s}^{*}}{\sqrt{2 \pi \nu^{* 2}}} \exp \left[\frac{-\left(t^{*}-t_{i}^{*}\right)^{2}}{2 \nu^{* 2}}\right] .
$$

The stimulation occurs at time $t_{i}^{*}$ for a duration $\nu$ and at amplitude $\kappa_{s}^{*}$. Subsequent active TGF- $\beta$ signalling pathways drive further mechanical activation of latent TGF- $\beta$ via ASM contraction and is assumed to occur at rate $\hat{\kappa}_{a c}^{*}\left(a^{*}, c^{*}\right)$, as in (4). Active TGF- $\beta$ is a contractile agonist and therefore generates a positive feedback loop of further TGF- $\beta$ activation. As reflected in (4), we assume that this process saturates so that the amount of achievable contraction of the ASM is physically limited. In addition to the natural degradation of active TGF- $\beta, \phi_{a}^{*}$, we must account for removal of active TGF- $\beta$ through binding to the receptors presented on both the proliferative and contractile ASM cells. Hence, we include the degradation term

$$
\hat{\kappa}_{b}^{*}\left(a^{*}, p^{*}, c^{*}\right)=\kappa_{b}^{*} a^{*}\left(p^{*}+c^{*}\right),
$$

that describes the rate of change in concentration of active TGF- $\beta$.

Our model (1) is subject to the initial conditions:

$$
p^{*}(0)=P^{*}, \quad c^{*}(0)=C^{*}, \quad a^{*}(0)=A^{*}, \quad m^{*}(0)=M^{*},
$$

where $P^{*}, C^{*}, A^{*}, M^{*}>0$.

\subsection{Parameter choices}

There is a lack of detailed experimental data to appropriately fit the vast number of parameters in our proposed model (1). For example, the study of Bai et al. (2000) quantifies ASM mass evolution; however, this may not be entirely due to proliferation. Moreover, it is not clear whether parameters measured from in vitro experiments are an accurate reflection of those parameters in vivo. Therefore, we follow Baker et al. (2017) and choose an illustrative parameter set whereby comparable processes have similar rates. Where possible, we determine estimates for the order of magnitude of rates from the literature and personal communication with Dr Amanda L. Tatler. Baseline parameter values are given in Table 2 in Appendix A.

\subsection{Non-dimensionalisation}

The rate of natural degradation of active $\mathrm{TGF}-\beta, \phi_{a}^{*}$, is one of the few known rates within the model and occurs on the order of minutes (Wakefield et al., 1990; Makinde et al., 2007). Hence, we non-dimensionalise time, $t^{*}$, relative to $\phi_{a}^{*}$ such that

$$
t=t^{*} \phi_{a}^{*}
$$

We non-dimensionalise the density of proliferating ASM, $p^{*}$, contractile ASM, $c^{*}$, ECM, $m^{*}$ and concentration of active TGF- $\beta, a^{*}$, relative to reference quantities, $p_{r}^{*}, c_{r}^{*}, m_{r}^{*}$ and $a_{r}^{*}$, respectively, to obtain

$$
p(t)=\frac{p^{*}\left(t^{*}\right)}{p_{r}^{*}}, \quad c(t)=\frac{c^{*}\left(t^{*}\right)}{c_{r}^{*}}, \quad a(t)=\frac{a^{*}\left(t^{*}\right)}{a_{r}^{*}}, \quad m(t)=\frac{m^{*}\left(t^{*}\right)}{m_{r}^{*}} .
$$


The dimensionless version of the ODE model (1) is given by

$$
\begin{aligned}
\frac{\mathrm{d} p}{\mathrm{~d} t}= & \kappa_{p} p\left(1-\frac{p}{p_{\text {max }}}\right)\left(1+\frac{\kappa_{a p}(a p)^{n_{p}}}{\tilde{\eta}_{a p}+(a p)^{n_{p}}}\right)+\frac{\kappa_{c p} \gamma_{c}}{\gamma_{p}} c-\kappa_{p c} p, \\
\frac{\mathrm{d} c}{\mathrm{~d} t}= & \frac{\kappa_{p c} \gamma_{p}}{\gamma_{c}} p-\kappa_{c p} c-\left(\phi_{c}-\frac{\phi_{c m} m^{n_{m}}}{\tilde{\eta}_{c m}+m^{n_{m}}}\right) c, \\
\frac{\mathrm{d} a}{\mathrm{~d} t}= & \kappa_{a}+\left(\frac{\kappa_{s}}{\nu} \exp \left[\frac{-\left(t-t_{i}\right)^{2}}{\nu^{2}}\right]+\frac{\kappa_{a c}(a c)^{n_{c}}}{\tilde{\eta}_{a c}+(a c)^{n_{c}}}\right) \frac{c m}{\gamma_{a}} \\
\frac{\mathrm{d} m}{\mathrm{~d} t}= & \quad-\kappa_{b}\left(\frac{\gamma_{p}}{\gamma_{c}} p+c\right) a-a, \\
& +\frac{\left.\kappa_{c m}+\frac{\kappa_{a c m}(a c)^{n_{c}}}{\tilde{\eta}_{a c m}+(a c)^{n_{c}}}\right) \gamma_{c} c+\left(\kappa_{p m}+\frac{\kappa_{a p m}(a p)^{n_{p}}}{\tilde{\eta}_{a p m}+(a p)^{n_{p}}}\right) \phi_{p} p}{\tilde{\eta}_{m} m,}
\end{aligned}
$$

wherein, the dimensionless parameters

$$
\gamma_{p}=\frac{p_{r}^{*}}{m_{r}^{*}}, \quad \gamma_{c}=\frac{c_{r}^{*}}{m_{r}^{*}}, \quad \gamma_{a}=\frac{a_{r}^{*}}{m_{r}^{*}},
$$

represent the ratio of the reference quantities of proliferating ASM, $p_{r}^{*}$, contractile ASM, $c_{r}^{*}$, and active TGF- $\beta, a_{r}^{*}$, to that of ECM, $m_{r}^{*}$, respectively. The non-dimensionalisation of the remaining parameters in the model (12) are provided in Table 1 in Appendix A.

The model (12) is subject to the dimensionless initial conditions:

$$
p(0)=P, \quad c(0)=C, \quad a(0)=A, \quad m(0)=M,
$$

where,

$$
P=\frac{P^{*}}{p_{r}^{*}}, \quad C=\frac{C^{*}}{c_{r}^{*}}, \quad A=\frac{A^{*}}{a_{r}^{*}}, \quad M=\frac{M^{*}}{m_{r}^{*}} .
$$

\section{Asymptotic reduction}

Following discussions with Dr Amanda L. Tatler, we identify the fast and slow time scales associated with rapid signalling pathways and gradually evolving downstream effects. The basal non-mechanical activation of TGF- $\beta, \kappa_{a}$, ECM aided survival of contractile ASM, $\phi_{c m}$, ECM secretion from the contractile ASM, $\kappa_{c m}$, ECM secretion from the contractile ASM induced by active TGF- $\beta, \kappa_{a c m}$, and ECM secretion from other surrounding cells, $\kappa_{e}$, act on a slow timescale of hours to days, compared to the remaining rates within the systems that act over the order of minutes. Therefore, we introduce a small parameter, $0<\varepsilon \ll 1$, and scale $\kappa_{a}, \phi_{c m}, \kappa_{c m}, \kappa_{a c m}$ and $\kappa_{e}$ such that

$$
\kappa_{a}=\varepsilon \tilde{\kappa}_{a}, \quad \phi_{c m}=\varepsilon \tilde{\phi}_{c m}, \quad \kappa_{c m}=\varepsilon \tilde{\kappa}_{c m}, \quad \kappa_{a c m}=\varepsilon \tilde{\kappa}_{a c m}, \quad \kappa_{e}=\varepsilon \tilde{\kappa}_{e} .
$$

We proceed by expanding each of the variables via asymptotic series in $\varepsilon$ such that

$$
\begin{aligned}
p(t) & =p^{(0)}(t)+\varepsilon p^{(1)}(t)+\mathcal{O}\left(\varepsilon^{2}\right), \\
c(t) & =c^{(0)}(t)+\varepsilon c^{(1)}(t)+\mathcal{O}\left(\varepsilon^{2}\right), \\
a(t) & =a^{(0)}(t)+\varepsilon a^{(1)}(t)+\mathcal{O}\left(\varepsilon^{2}\right), \\
m(t) & =m^{(0)}(t)+\varepsilon m^{(1)}(t)+\mathcal{O}\left(\varepsilon^{2}\right) .
\end{aligned}
$$

Hereafter, we consider first order saturation of the Hill functions within the model (12), i.e. $n_{p}, n_{c}$, $n_{m}, n=1$. Substituting the expansions (17) into the model (12), we obtain the respective leading order system. 
At $\mathcal{O}(1)$ the model (12) reads

$$
\begin{aligned}
\frac{\mathrm{d} p^{(0)}}{\mathrm{d} t}= & \kappa_{p} p^{(0)}\left(1-\frac{p^{(0)}}{p_{\max }}\right)\left(1+\frac{\kappa_{a p} a^{(0)} p^{(0)}}{\tilde{\eta}_{a p}+a^{(0)} p^{(0)}}\right) \\
& +\frac{\kappa_{c p} \gamma_{c}}{\gamma_{p}} c^{(0)}-\kappa_{p c} p^{(0)} \\
\frac{\mathrm{d} c^{(0)}}{\mathrm{d} t}= & \frac{\kappa_{p c} \gamma_{p}}{\gamma_{c}} p^{(0)}-\left(\kappa_{c p}+\phi_{c}\right) c^{(0)} \\
\frac{\mathrm{d} a^{(0)}}{\mathrm{d} t}= & \left(\frac{\kappa_{s}}{\nu} \exp \left[\frac{-\left(t-t_{i}\right)^{2}}{\nu^{2}}\right]+\frac{\kappa_{a c} a^{(0)} c^{(0)}}{\tilde{\eta}_{a c}+a^{(0)} c^{(0)}}\right) \frac{c^{(0)} m^{(0)}}{\gamma_{a}} \\
& \quad-\kappa_{b}\left(\frac{\gamma_{p} p^{(0)}}{\gamma_{c}}+c^{(0)}\right)-a^{(0)}, \\
\frac{\mathrm{d} m^{(0)}}{\mathrm{d} t}= & \left(\kappa_{p m}+\frac{\kappa_{a p m} a^{(0)} p^{(0)}}{\tilde{\eta}_{a p m}+a^{(0)} p^{(0)}}\right) \gamma_{p} p^{(0)}-\phi_{m} m^{(0)}
\end{aligned}
$$

\subsection{Reduced system steady states}

In the absence of stimulation, i.e. $\kappa_{s}=0$, there are four possible non-negative steady states of the reduced system (18). Hereafter, overbars indicate the steady state of each variable and subscripts denote each of the four steady state sets.

Firstly, in the leading order model (18), we identify the zero steady state

$$
\left(\bar{p}_{1}^{(0)}, \bar{c}_{1}^{(0)}, \bar{a}_{1}^{(0)}, \bar{m}_{1}^{(0)}\right)=(0,0,0,0) .
$$

Secondly, we identify a state where the leading order concentration of active TGF- $\beta$ is zero,

$$
\left(\bar{p}_{2}^{(0)}, \bar{c}_{2}^{(0)}, \bar{a}_{2}^{(0)}, \bar{m}_{2}^{(0)}\right)=\left(\bar{p}_{2}^{(0)}, \bar{c}_{2}^{(0)}, 0, \bar{m}_{2}^{(0)}\right),
$$

where,

$$
\begin{aligned}
\bar{p}_{2}^{(0)} & =p_{\max }\left(1-\frac{\kappa_{p c} \phi_{c}}{\kappa_{p}\left(\kappa_{c p}+\phi_{c}\right)}\right) \\
\bar{c}_{2}^{(0)} & =\frac{\kappa_{p c} \gamma_{p} \bar{p}_{2}^{(0)}}{\gamma_{c}\left(\kappa_{c p}+\phi_{c}\right)}, \\
\bar{m}_{2}^{(0)} & =\frac{\kappa_{p m} \gamma_{p} \bar{p}_{2}^{(0)}}{\phi_{m}} .
\end{aligned}
$$

The steady state (20) is positive and thus, biologically relevant, for

$$
\frac{\kappa_{p c} \phi_{c}}{\kappa_{p}\left(\phi_{c}+\kappa_{c p}\right)}<1 .
$$

Finally, we identify a further two positive steady states,

$$
\left(\bar{p}_{3,4}^{(0)}, \bar{c}_{3,4}^{(0)}, \bar{a}_{3,4}^{(0)}, \bar{m}_{3,4}^{(0)}\right)>(0,0,0,0),
$$

that are given by the intersection of the nullclines of the reduced system (18). The steady state 
solutions (23) are given by the intersections of the hypersurfaces:

$$
\begin{aligned}
\bar{p}_{3,4}^{(0)} & =p_{\max }\left(1-\frac{\kappa_{p c} \phi_{c}}{\kappa_{p}\left(\phi_{c}+\lambda_{p c}\right)}\left(\frac{\tilde{\eta}_{a p}+\bar{a}_{3,4}^{(0)} \bar{p}_{3,4}^{(0)}}{\tilde{\eta}_{a p}+\left(\kappa_{a p}+1\right) \bar{a}_{3,4}^{(0)} \bar{p}_{3,4}^{(0)}}\right)\right) \\
\bar{c}_{3,4}^{(0)} & =\frac{\kappa_{p c} \gamma_{p} \bar{p}_{3,4}^{(0)}}{\gamma_{c}\left(\kappa_{c p}+\phi_{c}\right)}, \\
\bar{a}_{3,4}^{(0)} & =\frac{\kappa_{p c}}{\kappa_{p c}+\kappa_{b}\left(\kappa_{c p}+\phi_{c}+\kappa_{p c}\right) \bar{c}_{3,4}^{(0)}}\left(\frac{\kappa_{a c} \bar{c}_{3,4}^{(0)} \bar{m}_{3,4}^{(0)}}{\gamma_{a}}\right. \\
\bar{m}_{3,4}^{(0)} & =\left(\kappa_{p m}+\frac{\kappa_{a p m} \bar{a}_{3,4}^{(0)} \bar{p}_{3,4}^{(0)}}{\tilde{\eta}_{a p m}+\bar{a}_{3,4}^{(0)} \bar{p}_{3,4}^{(0)}}\right) \frac{\kappa_{p c}}{\bar{c}_{3,4}^{(0)}}+\frac{\kappa_{b}\left(\kappa_{c p}+\phi_{c}\right.}{\phi_{m}}
\end{aligned}
$$

The system (24) reduces to two implicit equations for two of the states and hence, we obtain solutions numerically to confirm the existence of two positive states, $\bar{a}_{3}^{(0)}$ and $\bar{a}_{4}^{(0)}$, for a range of suitable parameter estimates.

\section{$4 \quad$ Stability analysis}

In the following, we determine the stability of the leading order zero steady state analytically (Section 4.1) and then proceed to perform a stability analysis of the fully nonlinear model (12) numerically (Section 4.2).

\subsection{Reduced system stability analysis}

The stability of the leading order steady states is determined by the eigenvalues of the corresponding Jacobian matrix of (18). Below, we consider the leading order zero steady state (19), for which eigenvalues can be obtained analytically. The Jacobian matrix for the reduced system (18) evaluated at (19), $\mathbf{J}_{0}$, is given by

$$
\mathbf{J}_{0}=\left[\begin{array}{cccc}
-\kappa_{p c}+\kappa_{p} & \frac{\kappa_{c p} \gamma_{c}}{\gamma_{p}} & 0 & 0 \\
\frac{\kappa_{p c} \gamma_{p}}{\gamma_{c}} & -\kappa_{c p}-\phi_{c} & 0 & 0 \\
0 & 0 & -1 & 0 \\
\kappa_{p m} \gamma_{p} & 0 & 0 & -\phi_{m}
\end{array}\right]
$$

Correspondingly, the eigenvalues, $\Lambda_{j}$ for $j \in\{1, \ldots, 4\}$, of $\mathbf{J}_{0}$ are given by

$$
\begin{aligned}
\Lambda_{1}= & -1, \\
\Lambda_{2}= & -\phi_{m}, \\
\Lambda_{3}= & \frac{\kappa_{p}-\kappa_{c p}-\kappa_{p c}-\phi_{c}}{2} \\
& \quad+\frac{1}{2}\left(\left(\kappa_{p}-\kappa_{c p}-\kappa_{p c}-\phi_{c}\right)^{2}-4\left(\phi_{c} \kappa_{p c}-\phi_{c} \kappa_{p}-\kappa_{c p} \kappa_{p}\right)\right)^{\frac{1}{2}}, \\
& \quad \frac{\kappa_{p}-\kappa_{c p}-\kappa_{p c}-\phi_{c}}{2} \\
& \quad-\frac{1}{2}\left(\left(\kappa_{p}-\kappa_{c p}-\kappa_{p c}-\phi_{c}\right)^{2}-4\left(\phi_{c} \kappa_{p c}-\phi_{c} \kappa_{p}-\kappa_{c p} \kappa_{p}\right)\right)^{\frac{1}{2}} .
\end{aligned}
$$

The eigenvalue $\Lambda_{3}$ is positive for

$$
\phi_{c} \kappa_{p c}-\phi_{c} \kappa_{p}-\kappa_{c p} \kappa_{p}<0
$$


and thus, for this parameter restriction, the leading order zero steady state (19) is unstable. The inequality (27) is equivalent to (22) and hence, this stability condition coincides with the existence of the biologically relevant steady state (20). The rate of ASM phenotype switching from the proliferative to contractile state, $\kappa_{p c}$, and the rate of ASM proliferation, $\kappa_{p}$, are of similar magnitude and thus (27) is satisfied for biologically relevant parameters. Accordingly, we have located a transcritical bifurcation in the leading order zero steady state (19).

\subsection{Full model stability analysis}

Guided by our findings at leading order in Section 4.1, we carry out a steady state stability analysis of the full model (12) in the dynamical systems software XPPAUT. The dimensionless parameters representing the rate of ASM proliferation, $\kappa_{p}$, phenotype switching from the proliferative to contractile phenotype, $\kappa_{p c}$ and contractile ASM degradation, $\phi_{c}$, (all relative to the rate of active TGF- $\beta$ degradation) feature in the reduced restriction (22). We therefore investigate the stability and location of the steady states of the full model (12) as functions of these parameters (Figure 2).

As previously identified in the reduced model (18), we observe regimes where there are up to four possible non-negative steady states, giving rise to a bi-stability (Figure 2). Within the chosen parameter ranges of $\kappa_{p}$ and $\kappa_{p c}$, we observe three distinct bifurcation points: a transcritical bifurcation, a change in stability of the positive branch and finally, another change in stability that gives rise to a bi-stable regime (see first two columns of Figure 2). The two points of stability change along the branch indicate the upper and lower bound of a bi-stable region. In the reduced model (18), these observations correspond to the transcritical bifurcation in the leading order zero steady state (19) as the leading order steady state (20) becomes positive and stable by satisfying the parameter restriction (22). The leading order steady states (23) correspond to the upper and lower bound of a bi-stable region.

As outlined in Section 1, there is a correlation in the severity of asthma and the amount of active TGF$\beta$ present in the airways (Chen and Khalil, 2006; Al Alawi et al., 2014). Within the bi-stable parameter regime, we associate the stable steady state where the concentration of active TGF- $\beta$ remains very small with a healthy homeostatic state that maintains normal ASM and ECM accumulation, and the stable steady state where the concentration of active TGF- $\beta$ is chronically elevated with a diseased state that results in excessive ASM and ECM accumulation. The unstable steady state located between the two acts as a transition threshold leading to irreversible airway alterations and asthma development. In the bifurcation diagrams in Figure 2, the healthy steady state is located in the lower section of the stable branch, the transition steady state is located in the unstable section of the branch and the diseased steady state is located in the upper stable section of the branch.

For values of $\kappa_{p}$ below the location of the transcritical bifurcation point, there are no biologically relevant steady states in the system (12) (e.g. Figure 2 (i)), suggesting that the proliferation rate must be large enough to allow for sustainable growth of the ASM and ECM populations. For increasingly large $\kappa_{p}$, the bi-stable regime is surpassed and the elevated positive steady state becomes the only biologically relevant steady state (e.g. Figure 2 (iv)). Intuitively, this steady state rises with increasing rate of ASM proliferation due to the accumulation of ASM which subsequently increases ECM secretion and causes further activation of TGF- $\beta$ (e.g. Figure 2 (vii)) that initiates a positive feedback loop.

On the other hand, there are no biologically relevant steady states in the system (12) for values of $\kappa_{p c}$ above the transcritical bifurcation point (e.g. Figure 2 (i)). This indicates that the ASM phenotype switching rate from the proliferative to the contractile state must be small enough so that there is a sufficient amount of ASM cells in the proliferative state in order to sustain ASM growth. This acts in a similar manner to the proliferation rate being too low, whereby ECM secretion and TGF- $\beta$ activation is hindered downstream. The stable steady state population of proliferating ASM increases as the switch rate continues to decrease; however, it is interesting to note that this corresponds to a decrease in the contractile ASM, ECM and active TGF- $\beta$ quantities once a maximum is achieved (e.g. Figure 2 (viii)). It appears that there is an optimal switching rate where there is sufficient proliferative ASM to increase growth and ECM secretion, yet allowing for a large contractile ASM 

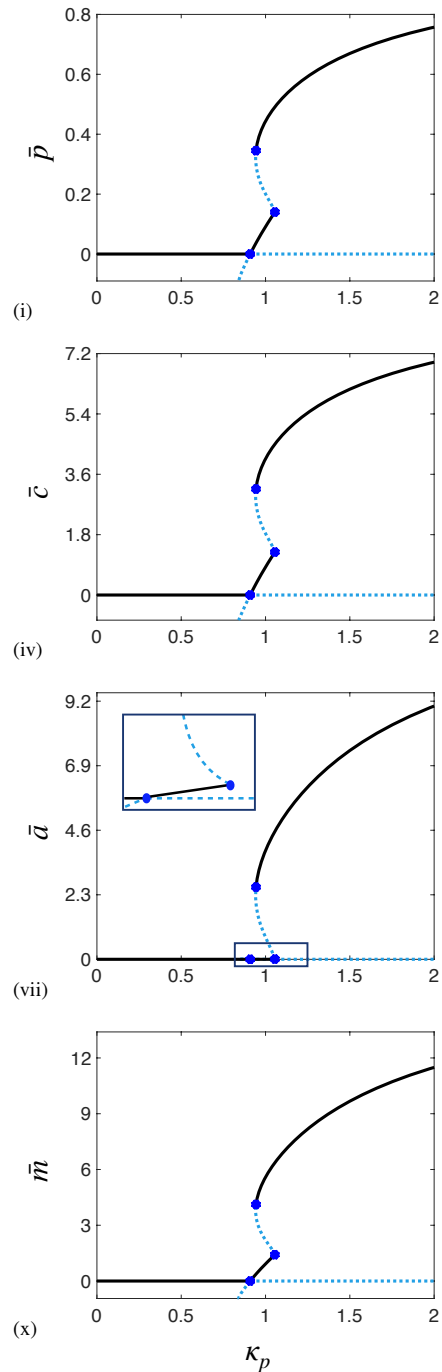

(ii)
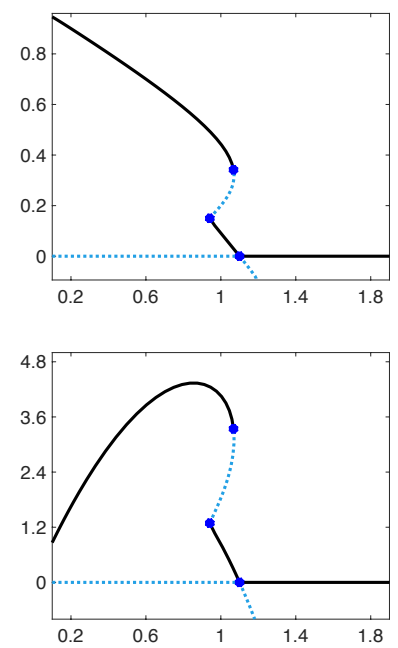

(v)

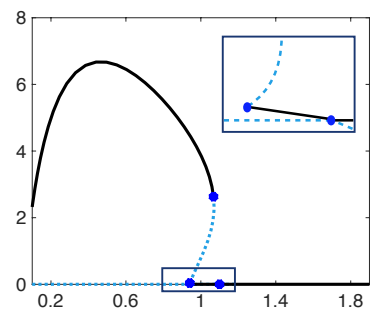

(viii)

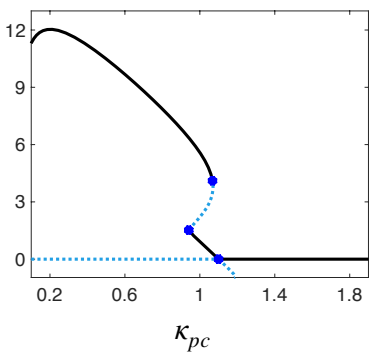

(iii)

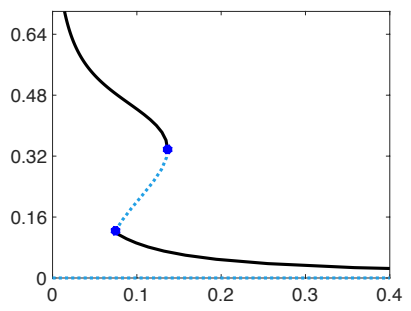

少

(vi)

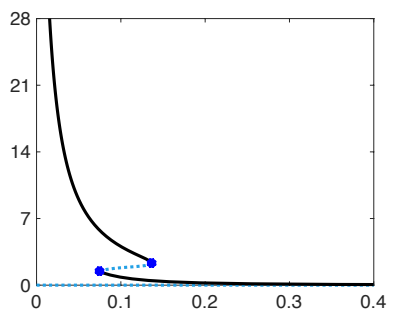

(ix)

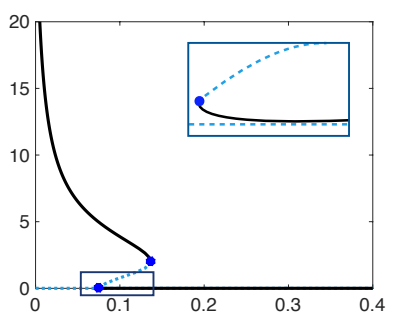

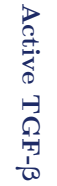

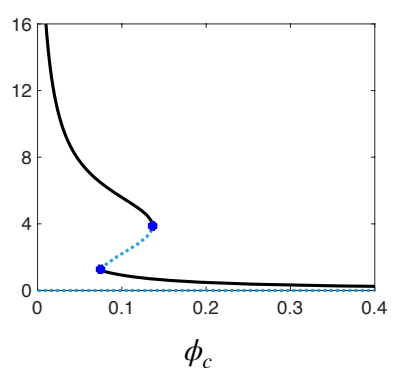

필

Figure 2: Bifurcation diagrams for $\kappa_{p}, \kappa_{p c}, \phi_{c}\left(1^{\text {st }}, 2^{\text {nd }}\right.$ and $3^{\text {rd }}$ column, respectively) in the model (12). The parameter values are baseline values provided in Table 2 in Appendix A.

population that is necessary to mechanically activate TGF- $\beta$ (Figure 2 (ii), (v)).

The two bifurcation points, where there is a change in direction and stability of the positive stable branch, persist as we vary the rate of degradation of the contractile ASM, $\phi_{c}$ (e.g. Figure 2 (iii)). For large values of $\phi_{c}$ there is a transcritical bifurcation, as observed in the other diagrams; however, we have restricted the range of $\phi_{c}$ that is displayed in the third column of Figure 2 in order to study the bi-stable regime of interest closely. Here, the lower region of the stable branch quickly reduces towards the the unstable steady state as $\phi_{c}$ increases. Whereas, there is a dramatic increase in the constituents' steady state value for very small values of $\phi_{c}$ (e.g. Figure 2 (vi)). In this instance, it is clear that the reduced rate of degradation of contractile ASM does not sufficiently regulate the accumulation of ASM mass and as a result, causes excessive growth of the contractile ASM, ECM secretion and TGF- $\beta$ activation (e.g. Figure 2 (xii)).

In Figure 3 we track the motion of the bifurcation points (shown in Figure 2) in two dimensional parameter space to show the creation and destruction of the bi-stable regime. Within the given parameter range, there exists a combination of $\kappa_{p}$ and $\kappa_{p c}$ that leads to a transcritical bifurcation point (Figure 3 (ii)). This point appears as both parameters increase, demonstrating a positive relation between the proliferation rate of ASM and the switching rate of ASM phenotype from the proliferative to contractile state. On the other hand, not all combinations with $\phi_{c}$ lead to a transcritical bifurcation point. In particular, for values of $\kappa_{p}>1$ and for values of $\kappa_{p c}<1$ we note that only the bi-stable 

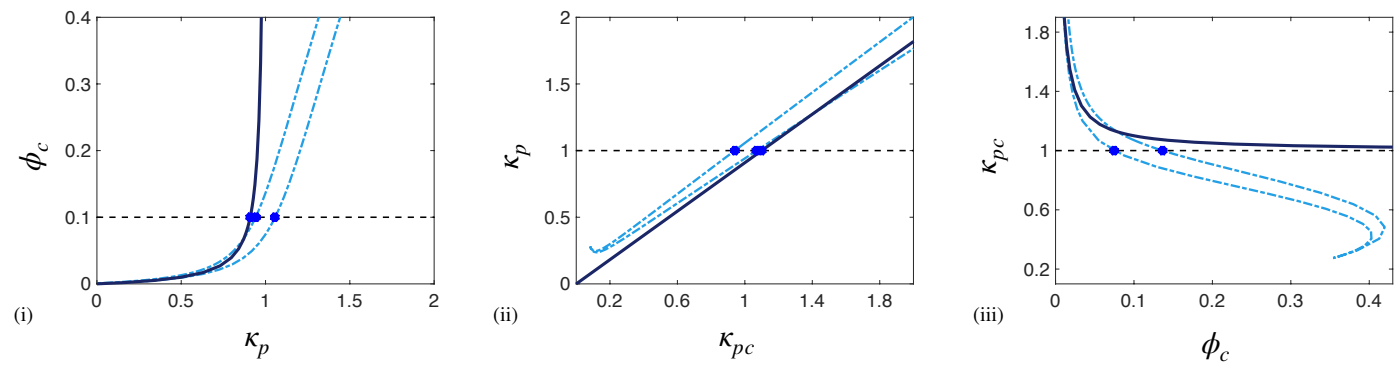

Figure 3: Two parameter bifurcation diagrams for $\kappa_{p}, \kappa_{p c}, \phi_{c}$ in the model (12). Solid lines track the transcritical branching point, blue dashed lines track the limit points defining the bi-stable regime, black dashed lines indicate a slice corresponding to Figure 2 and the blue dots represent the corresponding bifurcation points. The parameter values are baseline values provided in Table 2 in Appendix A.

regime exists (Figure 3 (i), (iii)). Therefore, a balance is required between the rate of proliferation and degradation of the ASM in order to permit a bi-stable regime that exhibits a state of relatively low concentration of active TGF- $\beta$. As proliferation increases, so must the degradation of ASM to regulate the increased ASM mass, subsequent ECM secretion and TGF- $\beta$ activation.

For very small values of $\kappa_{p c}$, we see that there are no bifurcation points for any value of $\phi_{c}$, meaning that there are no biologically relevant steady states in the system (Figure 3 (iii)). Therefore, when the ASM phenotype switching rate from the proliferative to contractile state is small, there are no rates of contractile ASM degradation that permit a biologically relevant steady state. In this instance, there is so little contractile ASM, due to insignificant amounts being switched from the proliferative population, that the degradation of what remains does not make a significant difference to the overall dynamics of the system.

Similarly, for very small values of $\kappa_{p}$, we see that not all values of $\kappa_{p c}$ permit a bi-stable regime and the transcritical bifurcation is the only bifurcation point in the system (Figure 3 (ii)). Biologically, the amount of proliferating ASM that may be exchanged to the contractile state is considerably reduced, due to the decline in the production rate. Hence, any minor contribution from the proliferative population is insignificant, irrespective of the switch rate, and does not alter the overall system dynamics.

\section{Numerical results}

As outlined above, the stable steady state where the concentration of active TGF- $\beta$ remains very small represents a healthy homeostatic state, and the stable steady state where the concentration of active TGF- $\beta$ is chronically elevated represents a diseased state. The unstable steady state located between the two acts as a transition threshold leading to irreversible airway alterations and asthma development. In this section, we demonstrate that in the presence of stimuli, $\hat{\kappa}_{s}(t)$ in $(7)$, the healthy homeostatic state may be surpassed, giving rise to the diseased state. Following the approach of Chernyavsky et al. (2014), here we simulate asthmatic exacerbations at multiple time points, $t_{i}$, via the dimensionless full model (12). We initialise the model (12) with the healthy homeostatic state and show the possible agonist-driven increase in the activation of TGF- $\beta$ and the downstream consequences that may lead to airway remodelling in asthma. All simulated parameter values, selected within the bi-stable regime, are provided in the relevant figure captions and in Table 3 in Appendix A.

The effect of increasing the frequency of recurrent challenges of contractile agonist is illustrated in Figure 4. For a low number of challenges, the system returns to the healthy homeostatic state following exposures (Figure 4 (i)). As the number of exposures increases, the concentration of active TGF- $\beta$ continues to climb (e.g. Figure 4 (ii)). Subsequently, ASM and ECM mass increases and leads to further activation of TGF- $\beta$ in a positive feedback loop. In this instance, the threshold for which the system can return to the healthy state is surpassed and the irreversible changes to the overall dynamics lead to the diseased state. Once a high number of successive exposures occur, ASM and 

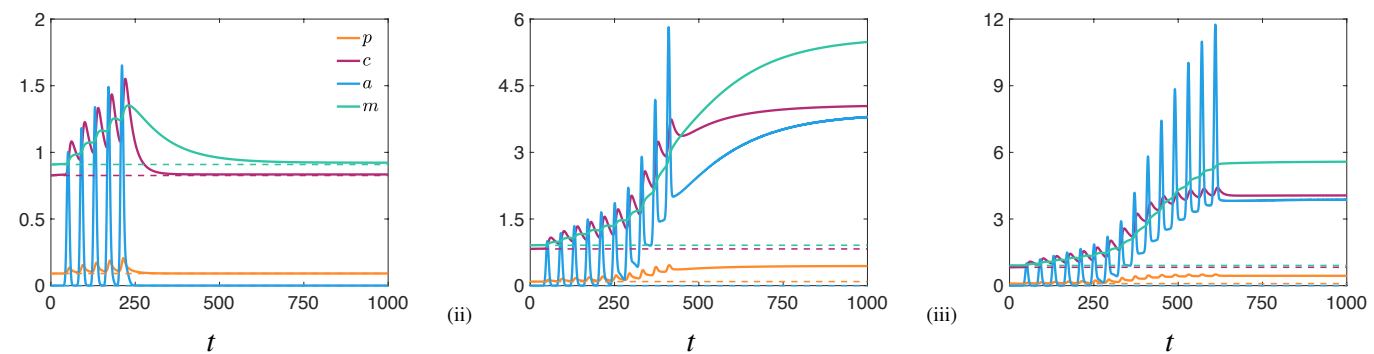

Figure 4: Results from the model (12) to illustrate the effect of increasing the number of stimuli (7) driving TGF- $\beta$ activation on the density of airway wall components. From (i)-(iii), the number of exposures are 5, 10 and 15. Dashed lines correspond to the healthy homeostatic state initial condition. Parameter values, selected within the bi-stable regime, are provided in Table 3 in Appendix A.

ECM accumulation appears to adapt to the newly adopted diseased regime and the relative amplitude of the perturbations decreases once a certain number of stimulations have been applied. The ASM mass, in both the proliferative and contractile forms, saturate at the diseased state and the relative amplitude of the perturbation from this state reduces at each exposure (Figure 4 (iii)).

The effect of reducing the period between exposures for a fixed number of recurrent stimuli is displayed in Figure 5. When the stimulation occurs at a low frequency there is sufficient recovery time between exposures for the system to return the healthy homeostatic state (Figure 5 (i)). Adequate clearance of active TGF- $\beta$ is hindered as the frequency increases (Figure 5 (iii)) and thus the underlying mechanotransductive feedback loop of further TGF- $\beta$ activation drives the system towards the diseased state. Removal of active TGF- $\beta$ via specified binding events in the model (12) sufficiently reduces the concentration of active TGF- $\beta$ which compensates for slightly more frequent exposures to the stimulant.

The effect of increasing the rate of clearance of active TGF- $\beta, \nu$, following exposure to a fixed number of recurrent stimuli, occurring at the same frequency, is shown in Figure 6. As previously in the case of increasing stimulus frequency (Figure 5), we find that as the clearance reduces, the concentration of active TGF- $\beta$ continues to rise and consequently re-establishes a new chronically elevated concentration. The persistent presence of active TGF- $\beta$, due to reduced clearance, affects the downstream dynamics and increases the amount of ASM and ECM (Figure 6 (i)). For moderate clearance, there is visible accumulation of ASM and ECM following the final exacerbation (Figure 6 (ii)). Interestingly, in the diseased cases, the model (12) demonstrates that the accumulation of ASM, ECM and active TGF- $\beta$ reduces below the diseased steady state following each exacerbation, subsequently increasing to attain the diseased state. This is evident for both slow clearance (Figure 6 (i)) and for moderate clearance (Figure 6 (ii)).
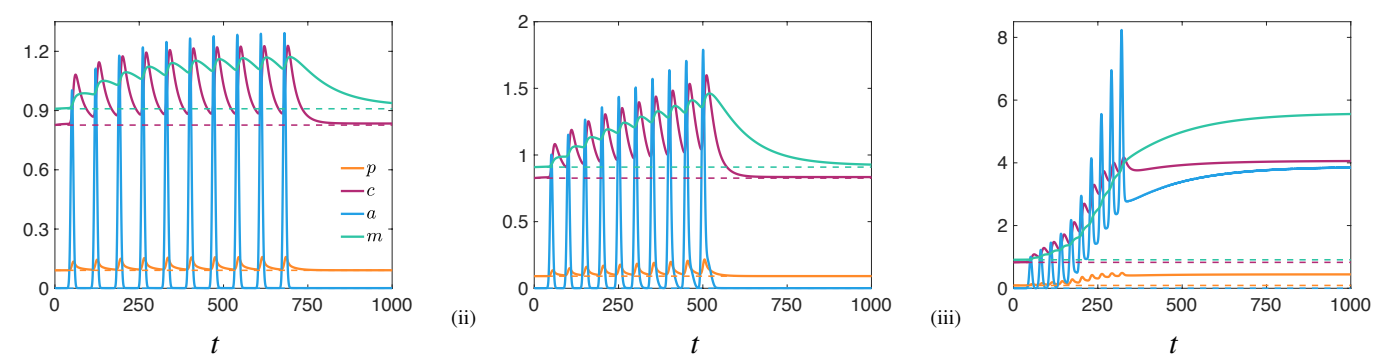

Figure 5: Results from the model (12) to illustrate the effect of increasing the frequency of stimuli (7) driving TGF- $\beta$ activation on the density of airway wall components. The frequency of exposures increase between plots from (i)-(iii). Dashed lines correspond to the healthy homeostatic state initial condition. Parameter values, selected within the bi-stable regime, are provided in Table 3 in Appendix A. 

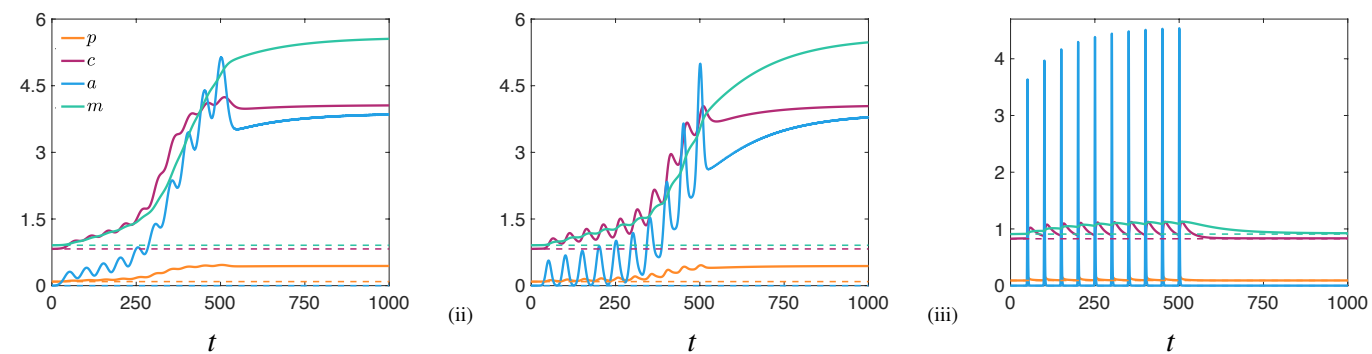

Figure 6: Results from the model (12) to illustrate the effect of increasing the clearance of active TGF- $\beta, \nu$ in (7), following stimuli, on the density of airway wall components. The value of $\omega$ increases between plots from (i)-(iii). Dashed lines correspond to the healthy homeostatic state initial condition. Parameter values, selected within the bi-stable regime, are provided in Table 3 in Appendix A.

A sufficient clearance rate allows for the system to return to the healthy homeostatic state in the model (12). The accumulation of ASM, ECM and active TGF- $\beta$ periodically rise and fall between the temporary peak and the healthy state following each exacerbation. Furthermore, the concentration of TGF- $\beta$ activated immediately following exposure to the stimulant is reduced compared to the disease free case in the simulations that investigate the effect of increasing the number and frequency of exposures (cf. Figures 6 (iii), 4 and 5). When the clearance of active TGF- $\beta$ is sufficient the system appears to adapt to experiencing challenges and returns to the healthy state following each exposure. Moreover, the temporary increase in active TGF- $\beta$ reaches a consistent maximum following exposure to the stimulant. This adaption process is similarly observed in the previous cases of the diseased state (e.g. Figure 4 (iii)).

The effect of increasing the amplitude of each exposure, $\kappa_{s}$, for a given number of recurrent stimuli, occurring at fixed frequency and clearance rate, is illustrated in Figure 7. As expected, we see that for relatively low amplitude stimulation, the long-term dynamics are unaffected by the temporary rise in ASM, ECM and active TGF- $\beta$ accumulation (Figure 7 (i)). Increasing $\kappa_{s}$ (Figure 7 (ii)), we observe a slight delay in the return from the temporary peak to the healthy steady state. This is highlighted by the accumulation of ASM and active TGF- $\beta$ (Figure 7 (ii)). Further increase gives rise to the disease state (Figure 7 (iii)). We find that the model is very sensitive to the strength of the stimulant and that very little change is required in order to significantly alter the downstream dynamics. Here, the percentage increase in $\kappa_{s}$ (the parameter representing the strength of the exacerbation) that is required to exhibit a significant change in the long-term behaviour is at least an order of magnitude smaller than in previous parameter explorations.
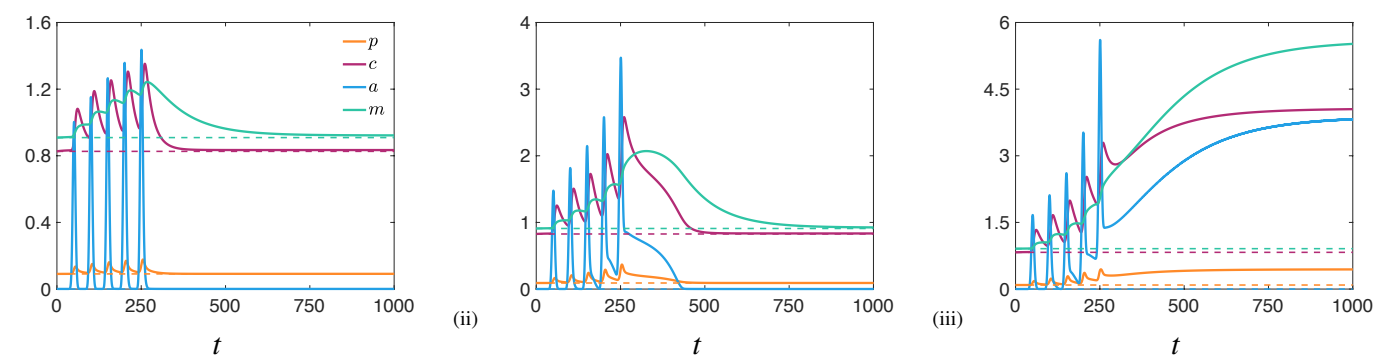

Figure 7: Results from the model (12) to illustrate the effect of increasing the strength of stimuli, $\kappa_{s}$ in (7), driving TGF- $\beta$ activation on the density of airway wall components. The value of $\kappa_{s}$ increases between plots from (i)-(iii). Dashed lines correspond to the healthy homeostatic state initial condition. Remaining parameter values, selected within the bi-stable regime, are provided in Table 3 in Appendix A. 


\section{Discussion}

In this study, we have presented a new model to describe the interaction of ASM and ECM and the effect of TGF- $\beta$ activation (accounting for active TGF- $\beta$ binding events) on the change in density of the airway wall constituents. We introduced suitable parameter scalings (based on estimates of timescales of interaction rates provided by Dr Amanda L. Tatler) via a small parameter, $\varepsilon$, that allowed us to reduce our model to a leading order system that permits some semi-analytical progress. Hereby, we determined the important parameters dominating homeostasis versus disease on which we then focused in a bifurcation analysis in the nonlinear model. This analysis exposes a bi-stable region in parameter space. This bi-stable regime allows for both a steady state maintaining a relatively low concentration of active TGF- $\beta$, representing healthy homeostasis, and a relatively higher concentration of active TGF- $\beta$ with accompanying increased ASM and ECM accumulation, representing diseased homeostasis. Results obtained in our bifurcation analysis demonstrate that the rate of ASM proliferation, degradation and phenotype switching from proliferative to contractile state dominate the long-term dynamics of the system. The ASM is the link between each of these factors and thus, our findings suggest that the ASM, in both a contractile and proliferative state, is a key component within the airway wall and is responsible for maintaining growth, ECM secretion and TGF- $\beta$ mechanical activation. We have shown that a balance is required between these three important roles to allow for the overall system to exhibit a bi-stable regime.

Results obtained simulating the possible downstream effects of introducing an external stimulant, mimicking an asthmatic exacerbation, highlight the importance of the number, the strength, the frequency of occurrence and the clearance of active TGF- $\beta$ following the exacerbation. We have observed that both an increased frequency of exposures to a stimulant and a reduced rate of clearance of active TGF- $\beta$ following exposure increases the accumulation in ASM and ECM and alters the steady state of the system in the long-term. This may be apparent in patients who experience regular exposures to an irritant and as a result, develop long-term irreversible airway remodelling. We have shown that allowing adequate recovery time or improving recovery time (i.e. reduced frequency and sufficient active TGF- $\beta$ clearance) between successive exacerbations returns the healthy homeostatic state. Therefore, in theory, increasing the clearance of active TGF- $\beta$ could be a possible method of asthma management or treatment.

Our findings are consistent with Chernyavsky et al. (2014) and Hill et al. (2018), who likewise explore airway remodelling in response to transient inflammatory or contractile agonist challenges. Similarly, we find that when the clearance of active $\mathrm{TGF}-\beta$ is inefficient (i.e. the inflammation resolution speed is slow), a small number of exacerbation events may lead to significant airway remodelling that remains after the stimulant resides. Chernyavsky et al. (2014) consider remodelling due to the growth of the ASM alone (ASM hyperplasia) and that the phenotype switching rate from the contractile to proliferative state is modulated by the inflammatory status (which changes in response to the stimulant). In contrast, we model the accumulation of ECM and active TGF- $\beta$ accompanying ASM growth and consider a constant switch rate between the ASM sub-populations. In future work, we could consider a switch rate that is dependent upon the concentration of active TGF- $\beta$. Other considerations could include development towards a stochastic model and simulating likely outcomes due to exposures occurring at irregular intervals.

In this study, we found that the model is particularly sensitive to variation in $\kappa_{s}$, the parameter representing the strength of the asthmatic exacerbation. Therefore, parameterisation and thorough parameter sensitivity analyses form a natural extension of this work. Ideally, the model parameters would be fitted to experimental data, once such data is available.

The morphoelastic model of Hill et al. (2018) reveals persistent ASM contraction in asthmatics via either a mechanotransductive feedback loop, insufficient clearance of contractile agonists, or a combination of the two. Our ODE model uncovers a bi-stable regime and highlights a threshold concentration of active TGF- $\beta$ that once surpassed, leads to irreversible airway remodelling. Motivated by these findings, in forthcoming work we will couple subcellular signalling dynamics to nonlinear hyperelastic models of airway mechanics to study mechanochemical mechanisms underpinning asthma characteristics. 


\section{Acknowledgements}

Dr Hannah J. Pybus was supported by the London Mathematical Society Early Career Fellowship, Grant number: ECF-1920-49.

We thank Dr Amanda L. Tatler (Division of Respiratory Medicine, University of Nottingham, UK) for assisting with biological matters and providing advice regarding suitable model parameters.

\section{Appendix A Model parameters}

Tables of parameter values corresponding to the model (12) developed in Section 2.

Table 1: Table of parameters in the ODE model (1), including the dimension, biological description and the corresponding dimensionless parameters in the dimensionless model 12.

\begin{tabular}{|c|c|c|c|}
\hline Parameter & Dimension & Description & Dimensionless \\
\hline$p_{r}^{*}$ & $\frac{N}{L^{3}}$ & $\begin{array}{l}\text { Reference number of proliferating } \\
\text { ASM cells per unit volume of tissue. }\end{array}$ & \\
\hline$c_{r}^{*}$ & $\frac{N}{L^{3}}$ & $\begin{array}{l}\text { Reference number of contractile ASM } \\
\text { cells per unit volume of tissue. }\end{array}$ & \\
\hline$a_{r}^{*}$ & $\frac{N}{L^{3}}$ & $\begin{array}{l}\text { Reference number of active TGF- } \beta \\
\text { molecules per unit volume of tissue. }\end{array}$ & \\
\hline$m_{r}^{*}$ & $\frac{N}{L^{3}}$ & $\begin{array}{l}\text { Reference number of ECM proteins } \\
\text { per unit volume of tissue. }\end{array}$ & \\
\hline$p^{*}$ & $\frac{N}{L^{3}}$ & $\begin{array}{l}\text { Number of proliferating ASM cells per } \\
\text { unit volume of tissue. }\end{array}$ & $p=\frac{p^{*}}{p_{r}^{*}}$ \\
\hline$c^{*}$ & $\frac{N}{L^{3}}$ & $\begin{array}{l}\text { Number of contractile ASM cells per } \\
\text { unit volume of tissue. }\end{array}$ & $c=\frac{c^{*}}{c_{r}^{*}}$ \\
\hline$a^{*}$ & $\frac{N}{L^{3}}$ & $\begin{array}{l}\text { Number of active TGF- } \beta \text { molecules } \\
\text { per unit volume of tissue. }\end{array}$ & $a=\frac{a^{*}}{a_{r}^{*}}$ \\
\hline$m^{*}$ & $\frac{N}{L^{3}}$ & $\begin{array}{l}\text { Number of ECM proteins per unit vol- } \\
\text { ume of tissue. }\end{array}$ & $m=\frac{m^{*}}{m_{r}^{*}}$ \\
\hline$P^{*}$ & $\frac{N}{L^{3}}$ & $\begin{array}{l}\text { Initial number of proliferating ASM } \\
\text { cells per unit volume of tissue. }\end{array}$ & $P=\frac{P^{*}}{p_{r}^{*}}$ \\
\hline$C^{*}$ & $\frac{N}{L^{3}}$ & $\begin{array}{l}\text { Initial number of contractile ASM cells } \\
\text { per unit volume of tissue. }\end{array}$ & $C=\frac{C^{*}}{c_{r}^{*}}$ \\
\hline$A^{*}$ & $\frac{N}{L^{3}}$ & $\begin{array}{l}\text { Initial number of active TGF- } \beta \\
\text { molecules per unit volume of tissue. }\end{array}$ & $A=\frac{A^{*}}{a_{r}^{*}}$ \\
\hline$M^{*}$ & $\frac{N}{L^{3}}$ & $\begin{array}{l}\text { Initial number of ECM proteins per } \\
\text { unit volume of tissue. }\end{array}$ & $M=\frac{M^{*}}{m_{r}^{*}}$ \\
\hline$p_{\max }^{*}$ & $\frac{N}{L^{3}}$ & $\begin{array}{l}\text { Maximum number of proliferating } \\
\text { ASM cells per unit volume of tissue. }\end{array}$ & $p_{\max }=\frac{p_{\max }^{*}}{p_{r}^{*}}$ \\
\hline$\gamma_{a}$ & 1 & $\begin{array}{l}\text { Ratio of reference TGF- } \beta \text { concentra- } \\
\text { tion and reference ECM density. }\end{array}$ & $\gamma_{a}=\frac{a_{r}^{*}}{m_{r}^{*}}$ \\
\hline$\gamma_{p}$ & 1 & $\begin{array}{l}\text { Ratio of reference proliferating ASM } \\
\text { and reference ECM density. }\end{array}$ & $\gamma_{p}=\frac{p_{r}^{*}}{m_{r}^{*}}$ \\
\hline$\gamma_{c}$ & 1 & $\begin{array}{l}\text { Ratio of reference contractile ASM and } \\
\text { reference ECM density. }\end{array}$ & $\gamma_{c}=\frac{c_{r}^{*}}{m_{r}^{*}}$ \\
\hline$t^{*}$ & $\mathrm{~T}$ & Time. & $t=\phi_{a}^{*} t^{*}$ \\
\hline$t_{i}^{*}$ & $\mathrm{~T}$ & Time of stimulus. & $t_{i}=\phi_{a}^{*} t_{i}^{*}$ \\
\hline$\nu^{*}$ & $\mathrm{~T}$ & Duration of stimulus. & $\nu=\sqrt{2} \phi_{a}^{*} \nu^{*}$ \\
\hline$\phi_{a}^{*}$ & $\frac{1}{T}$ & Rate of active TGF- $\beta$ degradation. & $1=\frac{\phi_{a}^{*}}{\phi_{a}^{*}}$ \\
\hline$\phi_{c}^{*}$ & $\frac{1}{T}$ & Rate of contractile ASM apoptosis. & $\phi_{c}=\frac{\phi_{c}^{*}}{\phi_{a}^{*}}$ \\
\hline
\end{tabular}


Table 1: Table of parameters in the ODE model (1), including the dimension, biological description and the corresponding dimensionless parameters in the dimensionless model 12 (continued).

\begin{tabular}{|c|c|c|c|}
\hline Parameter & Dimension & Description & Dimensionless \\
\hline$\phi_{c m}^{*}$ & $\frac{1}{T}$ & $\begin{array}{l}\text { Rate of ECM aided contractile ASM } \\
\text { survival. }\end{array}$ & $\phi_{c m}=\frac{\phi_{c m}^{*}}{\phi_{a}^{*}}$ \\
\hline$\phi_{m}^{*}$ & $\frac{1}{T}$ & Rate of ECM degradation. & $\phi_{m}=\frac{\phi_{m}^{*}}{\phi_{a}^{*}}$ \\
\hline$\kappa_{p}^{*}$ & $\frac{1}{T}$ & Rate of ASM proliferation. & $\kappa_{p}=\frac{\kappa_{p}^{*}}{\phi_{a}^{*}}$ \\
\hline$\kappa_{a p}^{*}$ & 1 & $\begin{array}{l}\text { Rate of TGF- } \beta \text {-induced ASM prolifer- } \\
\text { ation. }\end{array}$ & $\kappa_{a p}=\kappa_{a p}^{*}$ \\
\hline$\kappa_{c p}^{*}$ & $\frac{1}{T}$ & $\begin{array}{l}\text { Rate of switch from contractile ASM } \\
\text { phenotype to proliferative ASM phe- } \\
\text { notype. }\end{array}$ & $\kappa_{c p}=\frac{\kappa_{c p}^{*}}{\phi_{a}^{*}}$ \\
\hline$\kappa_{p c}^{*}$ & $\frac{1}{T}$ & $\begin{array}{l}\text { Rate of switch from proliferative ASM } \\
\text { phenotype to contractile ASM pheno- } \\
\text { type. }\end{array}$ & $\kappa_{p c}=\frac{\kappa_{p c}^{*}}{\phi_{a}^{*}}$ \\
\hline$\kappa_{a}^{*}$ & $\frac{N}{L^{3} T}$ & Rate of basal TGF- $\beta$ activation. & $\kappa_{a}=\frac{\kappa_{a}^{*}}{\phi_{a}^{*} a_{r}^{*}}$ \\
\hline$\kappa_{s}^{*}$ & $\frac{L^{3}}{N}$ & $\begin{array}{l}\text { Rate of stimulus-induced TGF- } \beta \text { me- } \\
\text { chanical activation. }\end{array}$ & $\kappa_{s}=\frac{\kappa_{s}^{\alpha} c_{r}^{*}}{\sqrt{\pi}}$ \\
\hline$\kappa_{a c}^{*}$ & $\frac{L^{3}}{N T}$ & $\begin{array}{l}\text { Rate of TGF- } \beta \text {-induced TGF- } \beta \text { me- } \\
\text { chanical activation. }\end{array}$ & $\kappa_{a c}=\frac{\kappa_{a c}^{*} c_{r}^{*}}{\phi_{a}^{*}}$ \\
\hline$\kappa_{c m}^{*}$ & $\frac{1}{T}$ & $\begin{array}{l}\text { Rate of ECM secretion from contrac- } \\
\text { tile ASM. }\end{array}$ & $\kappa_{c m}=\frac{\kappa_{c m}^{*}}{\phi_{a}^{*}}$ \\
\hline$\kappa_{a c m}^{*}$ & $\frac{1}{T}$ & $\begin{array}{l}\text { Rate of TGF- } \beta \text {-induced ECM secre- } \\
\text { tion from contractile ASM. }\end{array}$ & $\kappa_{a c m}=\frac{\kappa_{a c m}^{*}}{\phi_{a}^{*}}$ \\
\hline$\kappa_{p m}^{*}$ & $\frac{1}{T}$ & $\begin{array}{l}\text { Rate of ECM secretion from prolifer- } \\
\text { ating ASM. }\end{array}$ & $\kappa_{p m}=\frac{\kappa_{p m}^{*}}{\phi_{a}^{*}}$ \\
\hline$\kappa_{a p m}^{*}$ & $\frac{1}{T}$ & $\begin{array}{l}\text { Rate of TGF- } \beta \text {-induced ECM secre- } \\
\text { tion from proliferating ASM. }\end{array}$ & $\kappa_{a p m}=\frac{\kappa_{a p m}^{*}}{\phi_{a}^{*}}$ \\
\hline$\kappa_{e}^{*}$ & $\frac{N}{L^{3} T}$ & $\begin{array}{l}\text { Rate of TGF- } \beta \text {-induced ECM deposi- } \\
\text { tion from alternative sources. }\end{array}$ & $\kappa_{e}=\frac{\kappa_{e}^{*}}{\phi_{a}^{*} m_{r}^{*}}$ \\
\hline$\kappa_{b}^{*}$ & $\frac{L^{3}}{N T}$ & $\begin{array}{l}\text { Rate of Active TGF- } \beta \text { binding to a } \\
\text { receptor presented on proliferating or } \\
\text { contractile ASM. }\end{array}$ & $\kappa_{b}=\frac{\kappa_{b}^{*} c_{r}^{*}}{\phi_{a}^{*}}$ \\
\hline$n_{p}$ & 1 & $\begin{array}{l}\text { Hill Coefficient for active TGF- } \beta \text { bind- } \\
\text { ing to proliferating ASM receptor. }\end{array}$ & $n_{p}$ \\
\hline$n_{c}$ & 1 & $\begin{array}{l}\text { Hill Coefficient for active TGF- } \beta \text { bind- } \\
\text { ing to contractile ASM receptor. }\end{array}$ & $n_{c}$ \\
\hline$n_{m}$ & 1 & $\begin{array}{l}\text { Hill Coefficient for ECM aided con- } \\
\text { tractile ASM survival. }\end{array}$ & $n_{m}$ \\
\hline$n$ & 1 & $\begin{array}{l}\text { Hill Coefficient for active TGF- } \beta \text { - } \\
\text { induced ECM deposition from alterna- } \\
\text { tive sources. }\end{array}$ & $n$ \\
\hline$\tilde{\eta}_{a p}^{*}$ & $\left(\frac{N^{2}}{L^{6}}\right)^{n_{p}}$ & Disassociation constant. & $\tilde{\eta}_{a p}=\frac{\tilde{\eta}_{a p}}{\left(a_{r}^{*} p_{x}^{*}\right)^{n p}}$ \\
\hline$\tilde{\eta}_{a p m}^{*}$ & $\left(\frac{N^{2}}{L^{6}}\right)^{n_{p}}$ & Disassociation constant. & $\tilde{\eta}_{a p m}=\frac{\eta^{*} a_{p m}}{\left(a_{x}^{*} p_{r}^{*}\right)^{n_{p}}}$ \\
\hline$\tilde{\eta}_{a c}^{*}$ & $\left(\frac{N^{2}}{L^{6}}\right)^{n_{c}}$ & Disassociation constant. & $\tilde{\eta}_{a c}=\frac{\tilde{\eta}_{a c}^{*}}{\left(a_{r}^{*} c_{\tilde{r}}^{*}\right)_{c}^{n_{c}}}$ \\
\hline$\tilde{\eta}_{a c m}^{*}$ & $\left(\frac{N^{2}}{L^{6}}\right)^{n_{c}}$ & Disassociation constant. & $\tilde{\eta}_{a c m}=\frac{\tilde{\eta}_{a c m}^{*}}{\left(a_{r}^{*} c_{r}^{*}\right)^{n_{c}}}$ \\
\hline$\tilde{\eta}_{c m}^{*}$ & $\left(\frac{N}{L^{3}}\right)^{n_{m}}$ & Disassociation constant. & $\tilde{\eta}_{c m}=\frac{\tilde{\eta}_{c m}^{*}}{\left(m_{r}^{*}\right)^{n} m}$ \\
\hline$\tilde{\eta}_{e}^{*}$ & $\left(\frac{N}{L^{3}}\right)^{n}$ & Disassociation constant. & $\tilde{\eta}_{e}=\frac{\tilde{\eta}_{e}^{*}}{\left(a_{r}^{*}\right)^{n}}$ \\
\hline
\end{tabular}


Table 2: Table of dimensionless baseline parameter values for the ODE model (12).

\begin{tabular}{llllll}
\hline Parameter & Baseline & Parameter & Baseline & Parameter & Baseline \\
\hline$t$ & 1000 & $p_{\max }$ & 1 & $n_{p}$ & 1 \\
$\phi_{c}$ & 0.1 & $\gamma_{p}$ & 1 & $n_{c}$ & 1 \\
$\phi_{m}$ & 0.01 & $\gamma_{a}$ & 0.06 & $n_{m}$ & 1 \\
$\phi_{c m}$ & 0.001 & $\gamma_{c}$ & 1 & $n$ & 1 \\
$\kappa_{p}$ & 1 & $\kappa_{a p}$ & 1 & $\tilde{\eta}_{a p}$ & 1 \\
$\kappa_{c p}$ & 0.01 & $\kappa_{p c}$ & 1 & $\tilde{\eta}_{a p m}$ & 10 \\
$\kappa_{a}$ & 0.001 & $\kappa_{a c}$ & 0.01 & $\tilde{\eta}_{a c}$ & 1 \\
$\kappa_{a p m}$ & 0.1 & $\kappa_{p m}$ & 0.1 & $\tilde{\eta}_{a c m}$ & 1 \\
$\kappa_{a c m}$ & 0.001 & $\kappa_{c m}$ & 0.0001 & $\tilde{\eta}_{c m}$ & 1 \\
$\kappa_{e}$ & 0.001 & $\kappa_{b}$ & 1 & $\tilde{\eta}_{e}$ & 1 \\
\hline
\end{tabular}

Table 3: Table of dimensionless parameter values for the ODE model (12) used to produce Figures $4-7$. The remaining parameter values are baseline values, as provided in Table 2 .

\section{Parameter}

\begin{tabular}{lccc} 
Figure 4 & $t_{i}$ & $\nu$ & $\kappa_{s}$ \\
\cline { 2 - 4 } (i) & $50: 40: 250$ & 5 & 0.1 \\
(ii) & $50: 40: 450$ & 5 & 0.1 \\
(iii) & $50: 40: 650$ & 5 & 0.1 \\
\hline
\end{tabular}

Figure 5

\begin{tabular}{lrcc} 
(i) & $50: 70: 750$ & 5 & 0.1 \\
(ii) & $50: 50: 550$ & 5 & 0.1 \\
(iii) & $50: 30: 350$ & 5 & 0.1 \\
\hline
\end{tabular}

Figure 6

$\begin{array}{llll}\text { (i) } & 50: 50: 550 & 20 & 0.1 \\ \text { (ii) } & 50: 50: 550 & 10 & 0.1 \\ \text { (iii) } & 50: 50: 550 & 1 & 0.1\end{array}$

Figure 7

$\begin{array}{llll}\text { (i) } & 50: 50: 300 & 5 & 0.1 \\ \text { (ii) } & 50: 50: 300 & 5 & 0.15 \\ \text { (iii) } & 50: 50: 300 & 5 & 0.17\end{array}$




\section{References}

M. Al Alawi, T. Hassan, and S. H. Chotirmall. Transforming growth factor $\beta$ and severe asthma: a perfect storm. Respiratory Medicine, 108:1409-1423, 2014.

Y. Amrani and R. A. Panettieri. Airway smooth muscle: contraction and beyond. The International Journal of Biochemistry \& Cell Biology, 35(3):272-276, 2003.

J. P. Annes, J. S. Munger, and D. B. Rifkin. Making sense of latent TGF- $\beta$ activation. Journal of Cell Science, 116(2), 2003.

Y. Aschner and G. P. Downey. Transforming growth factor- $\beta$ : master regulator of the respiratory system in health and disease. American Journal of Respiratory Cell and Molecular Biology, 54(5): 647-655, 2016.

R. K. Assoian, A. Komoriya, C. A. Meyers, D. M. Miller, and M. B. Sporn. Transforming growth factor- $\beta$ in human platelets. The Journal of biological chemistry, 258(11):7155-7160, 1983.

T. R. Bai, J. Cooper, T. Koelmeyer, P. D. Paré, and T. D. Weir. The effect of age and duration of disease on airway structure in fatal asthma. American Journal of Respiratory and Critical Care Medicine, 162:663-669, 2000.

M. Baker, B. S. Brook, and M. R. Owen. Mathematical modelling of cytokines, MMPs and fibronectin fragments in osteoarthritic cartilage. Journal of Mathematical Biology, 75(4):1-40, 2017.

J. K. Bentley and M. B. Hershenson. Airway smooth muscle growth in asthma: proliferation, hypertrophy, and migration. Proceedings of the American Thoracic Society, 5(1):89-96, 2008.

R. Berair, R. Saunders, and C. E. Brightling. Origins of increased airway smooth muscle mass in asthma. BMC Medicine, 11(145), 2013.

Y. Bossé, P. D. Paré, C. Y. Seow, and J. Hogg. Airway wall remodeling in asthma: from the epithelial layer to the adventitia. Current Allergy and Asthma Reports, 8:357-366, 2008.

C. E. Brightling, S. Gupta, S. Gonem, and S. Siddiqui. Lung damage and airway remodelling in severe asthma. Clinical \& Experimental Allergy, 42(5):638-649, 2012.

M. Brumen, A. S. Fajmut, A. Dobovišek, D. Dobovišek, and E. Roux. Mathematical modelling of Ca 2+ oscillations in airway smooth muscle cells. Journal of Biological Physics, 31:515-524, 2005.

J. K. Burgess, T. Mauad, G. Tjin, J. C. Karlsson, and G. Westergren Thorsson. The extracellular matrix - the under-recognised element in lung disease? The Journal of Pathology, 240(4):397-409, 2016.

L. Buscemi, D. Ramonet, F. Klingberg, A. Formey, J. Smith Clerc, J. J. Meister, and B. Hinz. The single-molecule mechanics of the latent TGF- $\beta 1$ complex. Current Biology, 21(24):2046-2054, 2011.

G. Chen and N. Khalil. TGF- $\beta 1$ increases proliferation of airway smooth muscle cells by phosphorylation of map kinases. Respiratory research, 7(1):2, 2006.

W. Cheng, K. Yan, L. Y. Xie, F. Chen, H. C. Yu, Y. X. Huang, and C. X. Dang. MiR-143-3p controls TGF- $\beta 1$-induced cell proliferation and extracellular matrix production in airway smooth muscle via negative regulation of the nuclear factor of activated T cells 1. Molecular immunology, 78:133-139, 2016.

I. L. Chernyavsky, H. Croisier, L. A. C. Chapman, L. S. Kimpton, J. E. Hiorns, B. S. Brook, O. E. Jensen, C. K. Billington, I. P. Hall, and S. R. Johnson. The role of inflammation resolution speed in airway smooth muscle mass accumulation in asthma: insight from a theoretical model. PLoS ONE, 9(3):1-10, 2014.

H. Croisier, X. Tan, J. F. Perez Zoghbi, M. J. Sanderson, J. Sneyd, and B. S. Brook. Activation of store-operated calcium entry in airway smooth muscle cells: insight from a mathematical model. PloS one, 8(7), 2013. 
R. Derynck and Y. E. Zhang. Smad-dependent and smad-independent pathways in TGF- $\beta$ family signalling. Nature, 425:577-584, 2003.

A. M. Freyer, S. R. Johnson, and I. P. Hall. Effects of growth factors and extracellular matrix on survival of human airway smooth muscle cells. American Journal of Respiratory Cell and Molecular Biology, 25:569-576, 2001.

P. E. Gleizes, J. S. Munger, I. Nunes, J. G. Harpel, R. Mazzieri, I. Noguera, and D. B. Rifkin. TGF- $\beta$ latency: biological significance and mechanisms of activation. Stem Cells, 15(3):190-197, 1997.

C. L. Grainge, L. C. K. Lau, J. A. Ward, V. Dulay, G. Lahiff, S. Wilson, S. Holgate, D. E. Davies, and P. H. Howarth. Effect of bronchoconstriction on airway remodeling in asthma. The New England Journal of Medicine, 364(21):2006-2015, 2011.

T. Haberichter, E. Roux, M. Marhl, and J. P. Mazat. The influence of different InsP 3 receptor isoforms on Ca 2 + signaling in tracheal smooth muscle cells. Bioelectrochemistry, 57:129-138, 2002.

M. R. Hill, C. J. Philp, C. K. Billington, A. L. Tatler, S. R. Johnson, R. D. O’Dea, and B. S. Brook. A theoretical model of inflammation- and mechanotransduction-driven asthmatic airway remodelling. Biomechanics and Modeling in Mechanobiology, 17(5):1451-1470, 2018.

B. Hinz. The extracellular matrix and transforming growth factor- $\beta 1$ : tale of a strained relationship. Matrix Biology, 47:54-65, 2015.

G. Ijpma, A. Panariti, A. M. Lauzon, and J. G. Martin. Directional preference of airway smooth muscle mass increase in human asthmatic airways. American Journal of Physiology - Lung Cellular and Molecular Physiology, 312(6):845-854, 2017.

A. Januskevicius, S. Vaitkiene, R. Gosens, I. Janulaityte, D. Hoppenot, R. Sakalauskas, K. Malakauskas, L. F. Keglowich, P. Borger, S. S. Possa, D. C. Doeing, J. Solway, M. L. Fajt, S. E. Wenzel, A. J. Halayko, S. Holgate, J. Bousquet, K. Kumawat, T. Koopmans, R. Gosens, B. Yeganeh, A. L. James, R. Halwani, K. Kumawat, P. R. A. Johnson, H. Clevers, C. Logan, R. Nusse, H. A. Baarsma, M. Konigshoff, R. Gosens, B. G. J. Dekkers, K. Kumawat, R. Gosens, S. R. White, N. Krug, J. Bousquet, J. K. Bentley, M. B. Hershenson, M. Kato, S. S. Possa, F. Taki, J. M. Hughes, S. Sur, J. Mattes, B. Lamkhioued, L. Lintomen, A. B. Kay, S. Phipps, D. S. Robinson, R. T. Moon, S. Sokol, D. F. Choy, and L. Liu. Eosinophils enhance WNT-5a and TGF- $\beta 1$ genes expression in airway smooth muscle cells and promote their proliferation by increased extracellular matrix proteins production in asthma. BMC Pulmonary Medicine, 16(1):94, 2016.

P. R. A. Johnson, M. Roth, M. Tamm, J. M. Hughes, Q. Ge, G. King, J. K. Burgess, and J. L. Black. Airway smooth muscle cell proliferation is increased in asthma. American Journal of Respiratory and Critical Care Medicine : An Official Journal of the American Thoracic Society, Medical Section of the American Lung Association, 164(3):474-477, 2001.

J. Keski-Oja, K. Koli, and H. von Melchner. TGF- $\beta$ activation by traction? Trends in Cell Biology, 14(12):657-659, 2004.

N. Khalil, Y. D. Xu, R. O'Connor, and V. Duronio. Proliferation of pulmonary interstitial fibroblasts is mediated by transforming growth factor- $\beta 1$-induced release of extracellular fibroblast growth factor-2 and phosphorylation of p38 MAPK and JNK. Journal of Biological Chemistry, 280(52): 43000-43009, 2005.

M. A. Khan. Dynamics of airway response in lung microsections: a tool for studying airway-extra cellular matrix interactions. Journal of Biomedical Science, 23(1):43, 2016.

G. G. King, P. D. Paré, and C. Y. Seow. The mechanics of exaggerated airway narrowing in asthma: The role of smooth muscle. Respiration Physiology, 118(1):1-13, 1999.

K. H. Kuo, A. M. Herrera, and C. Y. Seow. Ultrastructure of airway smooth muscle. Respiratory Physiology \& Neurobiology, 137(2-3):197-208, 2003. 
B. León. T cells in allergic asthma: key players beyond the Th2 pathway. Current Allergy and Asthma Reports, 17(7):43, 2017.

J. J. Letterio and A. B. Roberts. Regulation of Immune Responses by TGF- $\beta$. Annual Review of Immunology, 16:137-61, 1998.

T. Makinde, R. F. Murphy, and D. K. Agrawal. The regulatory role of TGF- $\beta$ in airway remodeling in asthma. Immunology and cell biology, 85(5):348-56, 2007.

C. A. Ojiaku, G. Cao, W. Zhu, E. J. Yoo, M. Shumyatcher, B. E. Himes, S. S. An, and R. A. Panettieri. TGF- $\beta 1$ evokes human airway smooth muscle cell shortening and hyperresponsiveness via smad3. American Journal of Respiratory Cell and Molecular Biology, 58(5):575-584, 2018.

G. Pelaia, T. Renda, L. Gallelli, A. Vatrella, M. T. Busceti, S. Agati, M. Caputi, M. Cazzola, R. Maselli, and S. A. Marsico. Molecular mechanisms underlying airway smooth muscle contraction and proliferation: implications for asthma. Respiratory Medicine, 102(8):1173-1181, 2008.

D.-W. Perng, Y.-C. Wu, K.-T. Chang, M.-T. Wu, Y.-C. Chiou, K.-C. Su, R.-P. Perng, and Y.-C. Lee. Leukotriene $\mathrm{C} 4$ induces TGF- $\beta 1$ production in airway epithelium via p38 kinase pathway. American Journal of Respiratory Cell and Molecular Biology, 34:101-107, 2006.

D. Pohlers, J. Brenmoehl, I. Löffler, C. K. Müller, C. Leipner, S. Schultze Mosgau, A. Stallmach, R. W. Kinne, and G. Wolf. TGF- $\beta$ and fibrosis in different organs - molecular pathway imprints. Biochimica et Biophysica Acta, 1792:746-756, 2009.

S. Saglani and C. M. Lloyd. Novel concepts in airway inflammation and remodelling in asthma. The European Respiratory Journal, 46(6):1796-804, 2015.

M. Shi, J. Zhu, R. Wang, X. Chen, L. Mi, T. Walz, and T. A. Springer. Latent TGF- $\beta$ structure and activation. Nature, 474(7351):343-351, 2011.

X. Tan and M. J. Sanderson. Bitter tasting compounds dilate airways by inhibiting airway smooth muscle calcium oscillations and calcium sensitivity. British Journal of Pharmacology, 171:646-662, 2014.

A. L. Tatler and G. Jenkins. TGF- $\beta$ activation and lung fibrosis. Annals of The American Thoracic Society, 9(3), 2012.

A. L. Tatler and G. Jenkins. Reducing affinity of $\alpha \mathrm{v} \beta 8$ interactions with latent TGF- $\beta$ : dialling down fibrosis. Annals of Translational Medicine, 3(1):8-10, 2015.

A. L. Tatler, A. E. John, L. Jolly, A. Habgood, J. Porte, C. E. Brightling, A. J. Knox, L. Pang, D. Sheppard, X. Huang, and G. Jenkins. Integrin $\alpha \mathrm{v} \beta 5$-mediated TGF- $\beta$ activation by airway smooth muscle cells in asthma. Journal of immunology (Baltimore, Md. : 1950), 187(11):60946107, 2011.

A. L. Tatler, A. T. Goodwin, O. Gbolahan, G. Saini, J. Porte, A. E. John, R. L. Clifford, S. M. Violette, P. H. Weinreb, H. Parfrey, P. J. Wolters, J. Gauldie, M. Kolb, and G. Jenkins. Amplification of TGF- $\beta$ induced ITGB6 gene transcription may promote pulmonary fibrosis. PloS one, 11(8): $0158047,2016$.

T. Trian, G. Benard, H. Begueret, R. Rossignol, P. O. Girodet, D. Ghosh, O. Ousova, J. M. Vernejoux, R. Marthan, J. M. Tunon de Lara, and P. Berger. Bronchial smooth muscle remodeling involves calcium-dependent enhanced mitochondrial biogenesis in asthma. The Journal of experimental medicine, 204(13):3173-81, 2007.

L. M. Wakefield, T. S. Winokur, R. S. Hollands, K. Christopherson, A. D. Levinson, and M. B. Sporn. Recombinant latent transforming growth factor $\beta$ - 1 has a longer plasma half-life in rats than active transforming growth factor $\beta-1$, and a different tissue distribution. The Journal of Clinical Investigation, 86(6):1976-1984, 1990. 
A. R. West, H. T. Syyong, S. Siddiqui, C. D. Pascoe, T. M. Murphy, H. Maarsingh, L. Deng, G. N. Maksym, and Y. Bossé. Airway contractility and remodeling: links to asthma symptoms. Pulmonary Pharmacology and Therapeutics, 26:3-12, 2013.

P. J. Wipff, D. B. Rifkin, J. J. Meister, and B. Hinz. Myofibroblast contraction activates latent TGF- $\beta 1$ from the extracellular matrix. The Journal of cell biology, 179(6):1311-23, 2007. 